\title{
STUDY AND COMPARISON OF VARIOUS COMMUNICATION BASED PROTECTIVE RELAYING SCHEMES FOR ELECTRICAL POWER TRANSMISSION SYSTEM
}

\author{
VarunChauhan $^{1}$ \\ ${ }^{1}$ Department of Electrical Engineering, DAV Institute of Engineering \& Technology, Kabir Nagar, Jalandhal44008, \\ Punjab, India
}

\begin{abstract}
The relay communication system is the heart of the protective scheme. The independent operation of the relays scheme for the protection of transmission line is not adequate in some cases where the time delay to clear faults beyond the zone1 reach for distance relays may be considered unacceptable. The need for additional intelligence in the protection of some transmission lines arises due to the failing of Distance protection. As the future may see more global the protection designs also require highbandwidth communications in order to achieve the required speed. In this paper there is the study and comparison of various communication based protective relaying schemes which utilize a communications path to send signals from the relaying system at one end of the line to that at the other end.
\end{abstract}

Key words: Wire Pilot, PLCC, Microwave channel

\section{INTRODUCTION}

The use of pilot relaying has grown over the years and is now in common use throughout the world. In some cases simple scheme of independent operation of relaying scheme for protection system is not sufficient. This is the case where the time delay to clear faults beyond the zone 1 reach for distance relays may be considered unacceptable. To overcome this situation we study and apply the protective relaying schemes which are based on a communications path such as pilot wire, PLCC and microwave channels to send signals from the relaying system at one end of the line to that at the other end.

Because the inadequacy of distance protection for reliable fault detection and selectivity. This occurs due to a number of reasons:

- Intermediate in feeds in multi terminal lines.

- Zero-sequence mutual coupling in parallel lines.

- Discontinuities due to series capacitors.

Due to this there is a problem in zone reach measurements that are variable and are not suitable for correct fault detection and selectivity

And hence there is a need for additional intelligence in the protection of some transmission lines. To provide high speed clearing of end-zone faults on a transmission line section, there must be some form of communication channel between the line section terminals. This communication or pilot channel compares fault conditions at the line terminals to determine whether the fault is internal or external. In this respect, pilot protection is an extension of differential protection.

There are several advantages to high speed simultaneous clearing of faults at all line terminals:

(a) Improved transient stability of the system.

(b) Reduced line damage and the possibility of conductor burn-down.

(c) Availability of high speed reclosing, for improved transient stability, minimum outage times, or low voltage conditions on portions of the system load.

The types of pilot channels available for protective relaying include:

(a)Wire-line (pilot-wire)

(b)Power-line carrier(P L C C)

(c) Microwave radio

(d)Fiber-optic pilot protection

\section{WIRE PILOT SYSTEMS}

Wire pilot relaying systems have been used for many years to protect transmission lines, but these schemes are limited in their application. Pilot relaying with a pilot wire requires an end-to-end metallic circuit over which $50-60-\mathrm{Hz}$ and $\mathrm{dc}$ voltage may be transmitted. Since the system involves transmission of a 50 or $60-\mathrm{Hz}$ wave replica in both magnitude and phase pilot wire protection is analogous to differential protection of busses transformers, and machines. The wire pilot pair is less than 9 miles $(14.5 \mathrm{~km})$ long. Wire pilot schemes are divided into two basic types: 
- Circulating current

- Opposed voltage

In a figure 2.1 shown below circulating current scheme for pilot protection a current balance relay is used at each end of the line. The currents shown in the figure represent the normal condition, with current entering one end of the line and leaving the other end. Under this condition, the CT secondary currents flow in the restraint windings of each relay and no current flows through the operate windings. If a fault occurs on the line, the currents at the right-hand end of the line will reverse, which will cause currents to flow in the operate windings of both relays.

The voltage balance system, or "opposed-voltage system" is shown in figure 2.2. In this system, the pilot wires are crossed and no current flows in the pilot wires under the normal condition shown in the figure, which forces the currents to flow through the restraint windings of each relay. Note that the role of the two windings has been reversed over that illustrated in the circulating current scheme. For both of the wire pilot schemes illustrated, only the currents of one phase are shown in the diagrams. If this concept were to be adopted, it would require three such systems, one for each phase, which increases the cost and complexity.

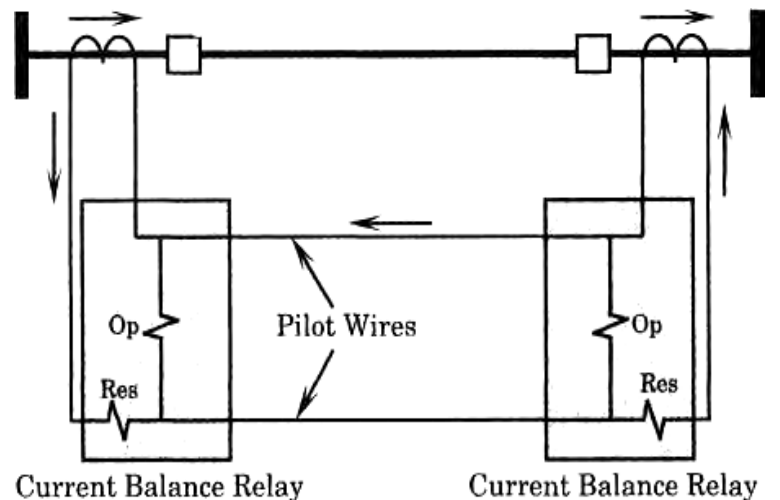

Current Balance Relay Current Balance Relay

Fig-1:Schematic of a circulating-current wire pilot system [3]

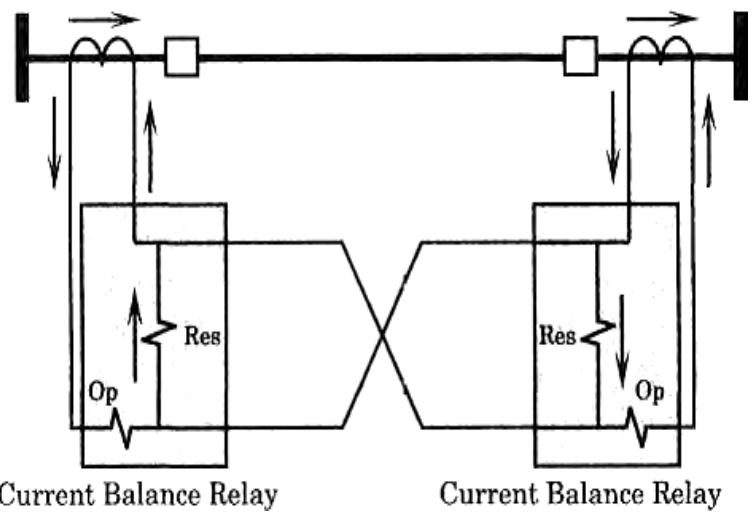

Fig-2:Schematic of a voltage balance wire pilot system [3]

\section{POWER LINE CARRIER CHANNELS}

Power line carrier has been applied to power lines in this country for over 80 years. In 1919, the first system for voice-communication purposes was placed into service. By the late twenties, power line carrier was used for pilot protective relaying, and has continued to provide this service ever since.[6]Carrier current of frequency range 30to 200 $(\mathrm{KHz})$ in USA and 80 to $500(\mathrm{KHz})$ in UK are transmitted and received through transmission line for the purpose of protection.[5]

\subsection{Frequency Range}

Power-line carrier generally operates over the band of frequencies between 30 and $300 \mathrm{kHz}$. These frequencies can be coupled to and transmitted over power lines with relatively small losses. Below $30 \mathrm{kHz}$, line coupling becomes impractical; above $300 \mathrm{kHz}$, the line losses are higher and radiation into space may interfere with licensed space radio services.

\subsection{Modes of Operation}

The power-line carrier signal can use on-off, frequency shift, or single-sideband (SSB) operation. The on-off signal is used to block tripping in an unfaulted line section; frequency-shift operation is used either for blocking or transfer-tripping. When functions are combined on an SSB channel audio tones are used to modulate the carrier, thus providing the relaying (and other) intelligence.

\subsection{Line Coupling}

The power-line phase conductor itself, a reliable physical circuit, is used to transmit the carrier signal between power stations. Most protective relaying carrier channels use single-phase-to-ground coupling, which has low attenuation (loss) and requires only one set of coupling equipment at a line terminal. Phase-to-phase coupling may be used to improve dependability. It offers a better chance of getting a signal through an internal fault when the channel is used for a transfer-trip relaying system.

\subsection{Insulated Static Wire}

Also applied as carrier channels, static wires have been used successfully for both voice communications and control functions. When static wires are used for carrier channels, the insulation level is usually around 7.5 to $15.0 \mathrm{kV}$. These insulation levels give rise to the possibility of insulator flash-over during a fault, which would greatly attenuate the carrier signal and prevent correct relay operation.[2]

\subsection{Major Carrier Components}

The major carrier components at one terminal of a line section equipped with a power-line carrier are shown in Figure3.5.1: 


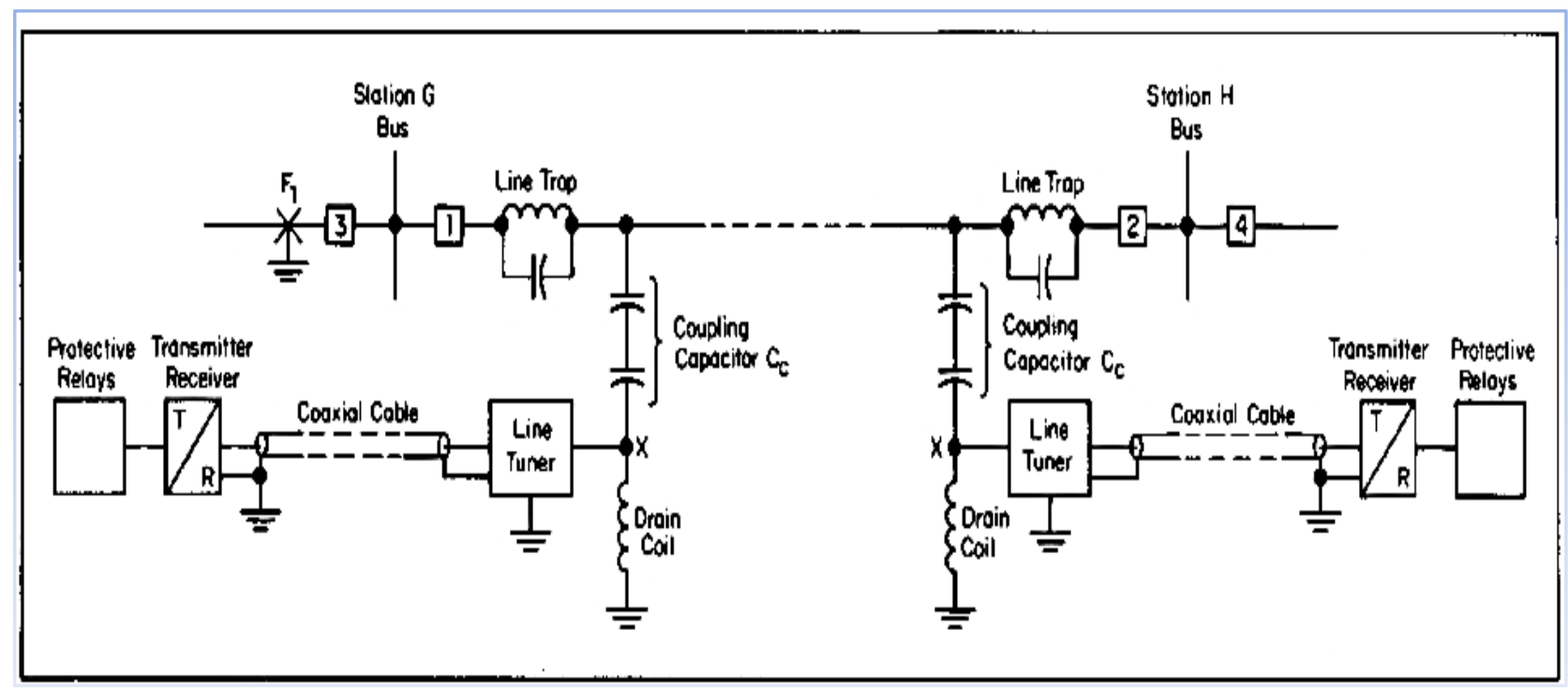

Fig-3.5.1:Basic Diagram of One Line Section Showing Main Carrier Components for Protective Relaying Channel

\subsubsection{Line Trap}

The line trap consists essentially of a parallel-tuned resonant circuit that presents high impedance to the operating carrier frequency but negligible impedance system frequency. The line trap serves two functions:

a. Prevents carrier energy from flowing into the station bus. Such a situation would reduce the signal in the desired direction and possibly cause interference in neighboring carrier channels.

b. Prevents an external ground fault behind the protective relays from short circuiting the carrier signal on the fault free line.A line trap must be able to carry the specified steady load current and fault current without overheating or suffering mechanical damage.

\subsubsection{Coupling Capacitor}

The carrier signals are coupled to the power line by the coupling capacitor, C". This unit, a series of stacked capacitors mounted inside Porcelain insulators, has a capacitance on the order depending on system ac voltage and potential burden requirements. Coupling capacitor allow the carrier frequency signal to enter the carrier equipment by providing low reactance to carrier frequency. To reduce impedance further a low inductance is connected in series with coupling capacitors to form a resonance at carrier frequency

\subsubsection{Drain Coil}

The drain coil, mounted in the base of the coupling capacitor, provides a low-impedance path for the flow of power-frequency current through the capacitor to ground, thus minimizing the system-frequency voltage developed from point $X$ to ground. At carrier frequencies, on the other hand, the drain coil has high impedance, minimizing $r-f$ losses to ground at this point.

\subsubsection{Line Tuner}

Applied in conjunction with the coupling capacitors, the line tuners provide an impedance match with low losses between the coaxial cable and the transmission line. Both resonant and broad-band line tuners are used. Two types of resonant tuners are available: single frequency and double frequency Figure 3.5.4.1. In the single frequency tuner Figure 3.5.4.1 (a), the inductive reactance of $\mathrm{L} 1$ cancels the capacitive reactance of the coupling capacitor $\mathrm{Cc}$ and isolating capacitor Cs at one frequency, thus providing a low-loss coupling circuit for the carrier frequency energy. The transformer $(\mathrm{Tl})$ matches the impedance of the coaxial cable to the surge impedance of the power line. The twofrequency resonant tuner Figure 3.5.4.1 (b)provides low-loss coupling at two separate frequencies from a single coaxial cable to the power line. The two-frequency tuner shown in Figure 3.5.4.1 (c) is used for isolating two different sets of carrier equipment. The path from each coaxial cable includes a matching transformer, ( $\mathrm{Tl}$ or $\mathrm{T} 2$ ) tuning inductance, and a trap circuit tuned to the other carrier frequency. In both cases, the isolating capacitor, Cs prevents the system-frequency current $(50$ or $60 \mathrm{~Hz})$ from the coupling capacitor from flowing through the tuning coil and matching transformer. Such a current flow might mask the relatively small carrier-frequency current. 


\subsubsection{Coaxial Cable}

Low-loss concentric cable is used to connect the line tuner and the carrier transmitter-receiver assemblies. Type RG8/U coaxial, recommended for this purpose, has a characteristic impedance of $52 \mathrm{ohms}$ and an attenuation of about 0.4 to 0.9 $\mathrm{dB} / 1000 \mathrm{Ft}$., increasing gradually over the 30 -to-300-kHz frequency range. The coaxial cable is generally grounded at the terminal end only to prevent system-frequency 50 or 60 $\mathrm{Hz}$ current from flowing over the coaxial cable shield during a ground fault. If both ends of the coaxial cable shield must be grounded to improve the shielding effect, a 4/0 copper cable should be run in the same duct to interconnect the ground points at the two ends of the coaxial cable. This arrangement will eliminate the possibility of current burning the coaxial cable shield open in the event of a ground fault.[2]

\section{MICROWAVE CHANNELS}

The microwave channel is a line-of-sight-radio system operating on a frequency band in the United States assigned by the Federal Communications Commission in the range from 950 to 30,000 megacycles. Such system requires that a straight line from one antenna to another antenna above intervening objects, preferably by about 50 foot. This usually limits the distance between antennae to about 20 to 50 miles, depending on the topography of the land [11].
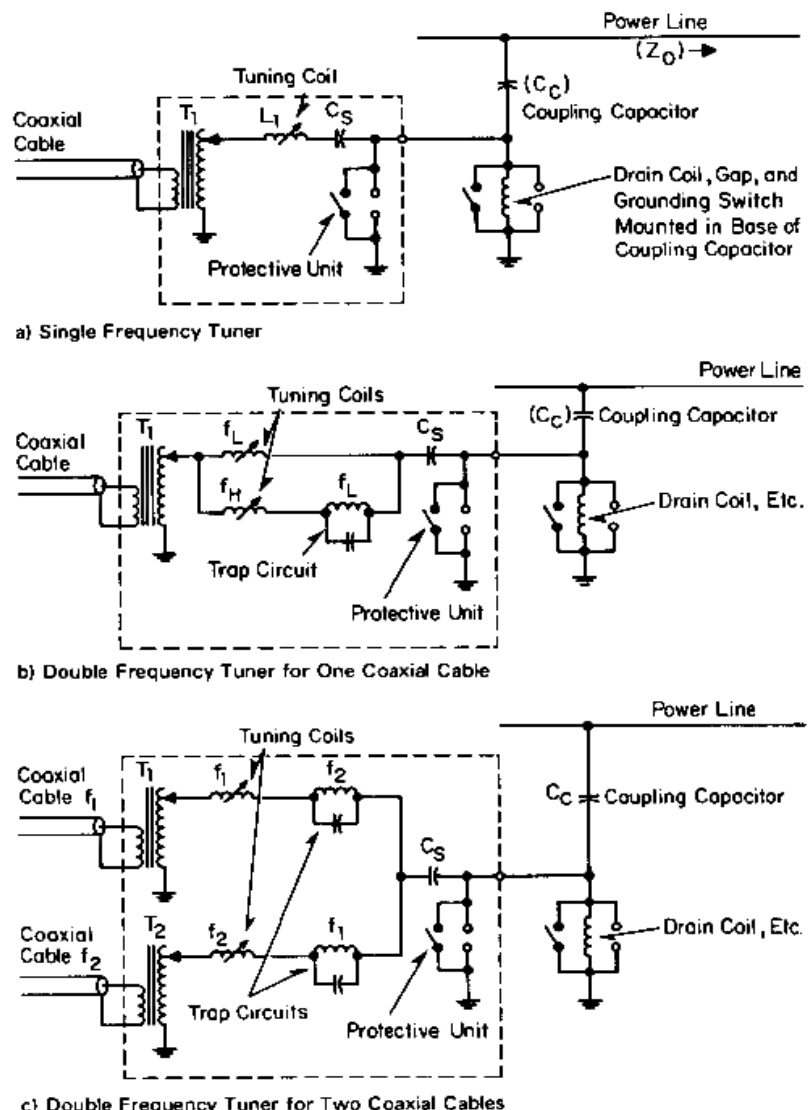

Fig-4:Schematic Diagram of Single and Double Frequency Resonant Line Tuners. [2.15.3]
Since the earth is spherical, a microwave beam that is ten miles from a point of tangency would be 66 feet above the earth's surface, and 264 feet above it when 20 miles away. Hence where a longer channel is required, one or more repeater stations may be necessary. One repeater station doubles the base channel equipment, except that only one additional tower is necessary; hence, the cost of a microwave channel is dependent on its length.

Microwave communication is used in the protection of long transmission lines where power line carrier does not offer enough channel capacity, or as a second communications path in addition to power line carrier. This is a radio channel employing very short wavelength or high frequency for point-to-point communication. Unlike power line carrier, the microwave signal is prorogated through the atmosphere between line-of-sight antenna locations. The basic microwave channel is subdivided or multiplexed so that it can be used for a large number of different functions at a same time.

When one of the sub-carrier channels of a microwave scheme is used for protective relaying, it is usually modulated by frequency shift audio tone equipment. The term "audio tone" as used here which is not restricted to a frequency audible to the human ear, but any frequency within the modulation capability of the microwave equipment. Audio tones in a nominal voice channel of 250 to 3,150 cycles per second can modulate the microwave frequency directly, or may be impressed on one of the multiplexed channels.

The number of microwave communicating channels which can be used is dependent on three factors:

1. Line-of-sight characteristic.

2. Angular directional characteristic.

3. Frequency selection.

\subsection{Advantages}

4.1.1.Channel reliability:. A microwave channel is reliable and is not dependent on the continuity of transmission line conductors or pilot wires, and is not subject to sleet, windstorm, or airplane damage.

4.1.2.Facilities per channel: The intelligence required by a large number of different functions can be transmitted on a single microwave channel using modulated subcarriers.

4.1.3. Freedom from interference.: Interference in the microwave band is very small and even may be a negligible factor.

4.1.4.Maintenance:Since the microwave channel is independent of the power system, maintenance can be performed without the necessity of having any part of the power system out of service. 


\subsection{Disadvantage}

4.2.1.Line-of-Sight:The necessity of line-of-sight will have a number of disadvantages; in some cases, it will necessitate repeater stations and the reliability of power supply at this repeater must be assured.

4.2.2.Power Supply: The high-voltage requirement necessitates the use of an AC power supply.

4.2.3.Structure Rigidity: There may be a problem of directivity caused by deflection of the antennas or mounting structures.

\subsection{Power Supply Considerations}

Microwave transmitters and receivers require higher tube plate voltage for satisfactory operation than is available from a station battery. For this reason, rectifier power supplies are used to supply the required direct voltage which is in the order of 300 to 500 volts, and in addition supply low alternating voltages for tube heater circuits. Such power supplies are usually energized from the 120 -volt 60 -cycle circuits which are available in the power station.

When a microwave channel is to be used for protective relaying, it is essential that there be no interruption of power which could allow incorrect operation of the relays. Such a requirement precludes the use of the local a-c source unless some provision is made to assure continuity of service even during fault conditions when such a source may be interrupted. Since the total power required for a microwave assembly is generally less than 1,000 watts, the motor and generator losses are negligible from an economic standpoint.[12]

\subsection{Design}

A frequency band of 6,575 to $6,875 \mathrm{mc}$ (megacycles) is used in the transmission. This selection was based on several theoretical considerations which made this band appear more suitable for protective relaying than the lower frequencies.

\subsubsection{Path Fading}

Although both high-frequency and low-frequency microwave bands suffer from approximately equal total propagation fading time, the duration of deep fading is very short for the higher frequency band. Deep fades are principally caused by 2-path propagation, reputedly the result of humidity inversion. The shorter wave lengths of the higher frequencies result in more rapid shifting of phase relationship between the beams at the receiving antenna, thus resulting in shorter fading periods.

\subsubsection{Antenna}

The short wave length permits the use of antennas at ground level and small high-gain passive reflectors, which can be located on structures or natural elevations, thus eliminating the need for coaxial cables with their high transmission losses, lightning hazards, and maintenance problems.

\subsubsection{Tubes}

Two low-power reflex klystrons are used in the generation and reception of the radio-frequency energy. These tubes are extremely reliable, having useful lives exceeding 10,000 hours. The transmitter klystron generates the radio frequency directly and is directly frequency modulated; therefore, no frequency multipliers are required.[9]

\subsection{Practices as Reported in Survey}

The results of the survey indicate that 8 of the 33 companies using microwave for protective relaying employ a separate parallel channel over a separate route for protection against microwave failure. 4 of the companies employ a separate channel via power-line carrier while the other 4 use a separate channel on cable. Of these 8 users all consider the microwave the primary channel of protection. The telephone company leases its microwave facilities for protective relaying purposes to 19 percent of the companies in the United States that reported in the survey. The telephone company leases both microwave RF channel and protective relaying multiplex channels. In all cases the power company provides the protective relays. In order to serve the relaying needs of power companies in the New York State area, the New York Telephone Company has built a number of microwave stations which are extensions of their main backbone. The stations are located adjacent to power company $345-\mathrm{kV}$ substations. These stations operate in the 6-GHz band and use frequency diversity. [10]

\section{CONCLUSION}

A various communication schemes is used to link the trip circuits at the ends of a transmission line provided with any conventional protection technique, thus insuring the simultaneous tripping of both line ends for all fault positions. The relay communication system is the heart of the protective scheme. The widespread use of transmission line protection by the communication facilities which reliable and provide high speed clearing of end-zone faults on a transmission line section. There are no rigid rules for the choice of communications method for pilot relaying. There are some characteristics that can be stated as general guidelines. The wire pilot protective relaying is used for transmission line less than 9 miles $(14.5 \mathrm{~km})$ long. 2. Power line carrier is used where the transmission line is too long to consider wire pilot. Microwave communication is used in the protection of long transmission lines where there is inadequate carrier spectrum available for PLC. The contrast on the comparison given below summarize more characteristic by providing advantages and disadvantages of various communication relaying schemes 


\section{CONTRAST ON THE COMPARISON OF COMMUNICATION RELAYING SCHEMES.}

\subsection{Wire-line}

\subsubsection{Advantages}

Economical for short lines

Can be used for magnitude comparison

\subsubsection{Disadvantages}

Subject to many natural and manmade hazards

Requires extensively protective equipment

\subsection{Power line carrier}

\subsubsection{Advantages}

Reliable, since channel is power line itself

Much less susceptible to natural or man-made hazard

Economical for Power lines too long for wire-line channel.

Convenient maintenance at line terminals

Economical for a small number of channels over a moderately long distance

Channel can extend through several line sections for control functions

Relaying carrier may include maintenance voice channel

\subsubsection{Disadvantages}

Limited frequency spectrum available in some areas Susceptible to power-line noise in some cases Line-voltage coupling and current-level traps required

\subsection{Microwave}

\subsubsection{Advantages}

Not affected by Power line noise.

Can handle a large number of voice and control sub channels over one microwave channels

Power line does not have to be taken out of service for terminal equipment maintenance.

\subsubsection{Disadvantages}

Initial installation expensive

Requires line-of-sight path which may require additional land

Path attenuation affected by weather, which may cause severe fading.

Hot stand by set often supplied, since many functions are dependent on common equipment.

\section{REFERENCES}

[1]J.H. Neher:“"The Use of Communication Facilitiesin Transmission Line Relaying”,AIEEE Transactions, Volume: 52, Issue: 2, Publication Year: 1933, Page(s): 595 - 602
[2] "Applied protective relaying”, Westinghouse Electric Corporation Relay Instrument Division Newark, N.J.O71O1457 pages.

[3]Paul M. Anderson: "Power System Protection", Volume 4 of IEEE Press Series on Power Engineering,Publisher Wiley, 19981330 pages.

[4]T. S. M. Rao: "Power System Protection: Static Relays", Edition 2, Reprint, Revised, Publisher Tata McGraw-Hill Education, 1989723 pages.

[5]Sunil S. Rao: "Switchgear Protection and Power Systems", Edition 11 KhannaPublishers, 19991691 pages.

[6]"Special considerations in applying power line carrier for protective relaying", IEEE Power systems relaying committee special paper, Relayingcommunications subcommittee, working group H9, 1-5-04

[7]J. R. Linders, V. A. Nosko: “An Approach to Microwave Relaying and System Design”, vol. 76, pt. III, Apr. 1957, pp. 19-27.

[8]"Microwave Channels for Power System Applications", AIEE Committee Report. AIEEE Transactions, volume 68, part I, 1949, pages 40-43.

[9]E. W. Downer: "Field Tests of Microwave Relaying Equipment",AIEEE Transactions, vol.76, no.3, pp.74,79, April 1957.

[10]"Use of Microwave Radio for Protective Relaying Power Apparatus and Systems", IEEE Transactions on Volume: PAS-89, Issue: 5 Publication Years: 1970, Page(s): $828-836$.

[11]GE Digital Energy: "Line Protection with Pilot Relays", art15, 322-346 pages.

[12]H W Lensner: "Protective relaying systems using microwave channels",Electrical Engineering, vol.71, no.5, pp.400-405, May 1952.

[13]Y.G. Paithankar, S.R. Bhide: "Fundamentals of Power System Protection", Edition 2, Publisher PHI Learning Pvt. Ltd., 2010 Length 366 pages

[14]Power System Protection: "Principles and Components", edited by The Electricity Training Association, Institution of Electrical Engineers, Edition2, llustrated, reprint Publisher IET, 1995, Length 554 pages.

[15]Sanders, M.P: "Narrowband power-line carrier for use in protective relaying applications"'Developments in Power System Protection, 2004. Eighth IEE International Conference on, vol.2, no., pp.660-664. 


\section{BIOGRAPHIE}

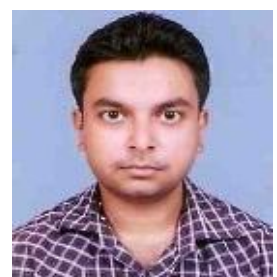

My name is Varun Chauhan completed B.Tech in Electrical Engineering from MMEC Mullana, Ambala affiliated to Kurukshetra University and know doing M.Tech in Electrical Engineering from DAVIET affiliated to PTU, Jalandhar. I have been worked in Elin Appliances Private Limited (Philips), District Solan in Production Department and also in E-max Institute District Ambala as Lecturer in Electrical Department. 